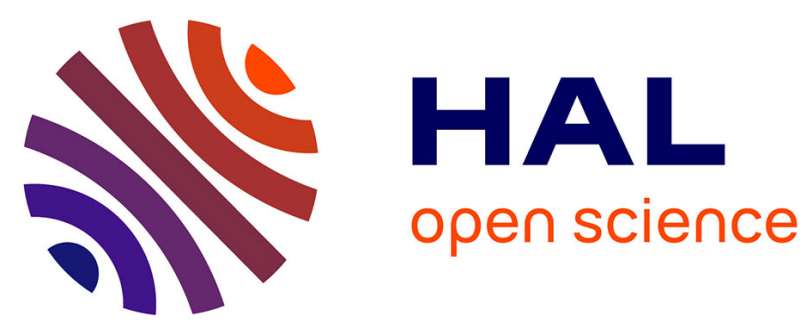

\title{
Le climat, un facteur de risque pour la santé en Afrique de l'Ouest.
}

\author{
Nadège Martiny, Nadine Dessay, Pascal Yaka, Ousmane Toure, Benjamin \\ Sultan, Stanislas Rebaudet, Hélène Broutin, Renaud Piarroux, Isabelle \\ Chiapello, Issaka Sagara, et al.
}

\section{To cite this version:}

Nadège Martiny, Nadine Dessay, Pascal Yaka, Ousmane Toure, Benjamin Sultan, et al.. Le climat, un facteur de risque pour la santé en Afrique de l'Ouest.. La Météorologie, 2012, Spécial AMMA, pp.73-79. 10.4267/2042/48135 . hal-00757107

\section{HAL Id: hal-00757107 \\ https://hal.science/hal-00757107}

Submitted on 28 Apr 2016

HAL is a multi-disciplinary open access archive for the deposit and dissemination of scientific research documents, whether they are published or not. The documents may come from teaching and research institutions in France or abroad, or from public or private research centers.
L'archive ouverte pluridisciplinaire HAL, est destinée au dépôt et à la diffusion de documents scientifiques de niveau recherche, publiés ou non, émanant des établissements d'enseignement et de recherche français ou étrangers, des laboratoires publics ou privés.

\section{(ㅇ)(1) $\$$}

Distributed under a Creative Commons Attribution - NonCommercial - NoDerivatives| 4.0 


\title{
Le climat, un facteur de risque pour la santé en Afrique de l'Ouest
}

\author{
Nadège Martiny ${ }^{(1)}$, Nadine Dessay ${ }^{(2)}$, Pascal Yaka ${ }^{(3)}$, Ousmane Toure ${ }^{(4)}$, \\ Benjamin Sultan $^{(5)}$, Stanislas Rebaudet ${ }^{(6)}$, Hélène Broutin ${ }^{(7)}$, \\ Renaud Piarroux $^{(6)}$, Isabelle Chiapello ${ }^{(8)}$, Issaka Sagara ${ }^{(4)}$, \\ Bernard Fontaine $^{(1)}$, Mahamadou Sissoko ${ }^{(4)}$, Isabelle Jeanne ${ }^{(9)}$, \\ Ogobara Doumbo ${ }^{(4)}$ et Jean Gaudart ${ }^{(10)}$ \\ (1) Université de Bourgogne, Centre de recherches de climatologie, \\ UMR CNRS 6282 (Biogéosciences), Dijon, France \\ ( 2) IRD, UMR 228 (ESPACE-DEV), Montpellier, France \\ ( 3 ) Direction générale de la météorologie du Burkina Faso, Ouagadougou, \\ Burkina Faso \\ (4) Université des Sciences, des techniques et technologies de Bamako, \\ Malaria Research and Training Center/DEAP, UMI-3189, Bamako, Mali \\ ( 5) Université Pierre et Marie Curie, UMR LOCEAN 7159, Paris, France \\ (6) Aix-Marseille Univ, UMR MD3, Marseille, France \\ ( 7) MIVEGEC, UMR 5290 CNRS- 224 IRD- UM1-UM2, Montpellier, France \\ ( 8) Université de Lille 1, UMR LOA 8518, Villeneuve d'Ascq, France \\ ( 9) Consultante internationale, Geelong, Australie \\ (10) Aix-Marseille Univ, UMR 912 (SESSTIM), Marseille, France.
}

Cet article fait le point sur certaines connaissances acquises dans le cadre du programme AMMA (phase 1) au sujet des impacts de la variabilité climatique sur la santé en Afrique de l'Ouest. Les auteurs ont travaillé d'une part sur les maladies sévissant majoritairement en saison des pluies, à vecteur tel que le paludisme à Plasmodium falciparum, ou non comme le choléra, et d'autre part sur les maladies respiratoires et non vectorielles, telles que les méningites bactériennes à méningocoques à Neisseira Meningitidis, qui sévissent en plein cour de la saison sèche. Le paludisme est connu pour sa relation à l'eau et à la température, indispensable pour le développement de son vecteur et son parasite. Le choléra, bien que lié à l'eau, peut aussi être observé en saison sèche : le lien entre choléra et environnement mérite donc un état des lieux éclairé par les nouveaux paramètres et outils disponibles aujourd'hui du côté du climat. L'intensité de la saison de la méningite est très liée aux conditions de vent et d'humidité prédominants en octobre-novembre de l'année précédente. Son démarrage est en phase avec le maximum de l'hiver et l'arrivée des grands événements de poussières dans les basses couches atmosphériques. Enfin, son arrêt est très lié à l'arrivée de la mousson. Ces résultats marquent une étape importante dans la mise en place de systèmes d'alerte précoce.

\section{Pourquoi l'étude des impacts du climat sur la santé s'est-elle révélée importante dans le cadre d'AMIMA?}

Le paludisme à Plasmodium falciparum est une maladie à transmission vectorielle parasitaire dont le lien avec le climat et l'environnement est bien connu. Son incidence est estimée entre 300 et 500 millions de nouveaux cas par an, dont $90 \%$ surviennent en Afrique subsaharienne (Breman et al., 2004 ; Snow et al., 2005; OMS, 2011). Son impact économique et social est non négligeable (Thuilliez et al., 2010) et il demeure l'une des premières causes de mortalité dans le monde. Cette maladie transmise par un moustique dont l'écologie est liée à l'eau, est souvent saisonnière dans la zone soudano-sahélienne. Cependant, l'échec, par le passé, des différentes politiques de lutte/ élimination rend toujours d'actualité la nécessité de mieux comprendre la relation entre le climat, l'homme, le vecteur et le parasite, à des échelles fines, afin d'adapter les actions au contexte local. Le choléra est généralement considéré comme le prototype des maladies liées à l'eau, en particulier du fait que Vibrio cholerae, l'agent du choléra, est d'abord un germe de l'environnement capable de se développer dans les eaux saumâtres des estuaires. Cette bactérie tolère aussi l'eau douce, surtout si la faible salinité est compensée par une chaleur importante et la présence de nutriments (Colwell, 1996). De nombreuses études ont mis en évidence le caractère saisonnier du choléra (Emch et al., 2008) et son incidence annuelle semble, dans certaines régions, guidée par les oscillations climatiques comme le phénomène El Niño (Pascual et al. 2000).

Les épidémies de méningite bactérienne sont principalement localisées dans la « ceinture de la méningite » (Lapeyssonnie, 1963) représentant une population de 400 millions de personnes. C'est un problème majeur de santé publique en Afrique de l'Ouest (OMS, 2000) qui est dû, en plus des facteurs immunitaires de la population humaine et des caractéristiques de virulence des bactéries, à la conjonction de facteurs sociaux (par exemple mouvements de populations ; Broutin et al., 2007), 
$\ldots / 4$

\section{Abstract}

\section{Climate as a risk factor for health in West Africa}

This article focuses on some knowledge gained in the AMMA program (Phase 1) on the impacts of climate variability on health in West Africa. The authors worked, on the one hand, on the diseases occurring mainly in the rainy season, vectorborne such as Plasmodium falciparum malaria, or not such as cholera, and, on the other hand, on respiratory and non vector-borne disease occurring in the heart of the dry season, such as Neisseira meningitidis bacterial meningitis. Malaria is known for its relationship to water and temperature, indispensable for the development of its vector and parasite. Cholera, linked to water, can also be observed in the dry season: the link between cholera and environment deserves an inventory illuminated by the new settings and tools available today on the side of the climate. The intensity of the meningitis season is related to wind conditions and humidity prevailing in October-November of the previous year. Its start is in phase with the winter maximum and the arrival of large dust events in the lower layers of the atmosphere. Finally, its end is linked to the arrival of the monsoon. These results mark an important step in the establishment of early warning systems. climatiques (conditions de sécheresse, etc. ; Greenwood et al., 1984) et environnementaux (exemple aérosols désertiques ; Molesworth et al., 2002). Les questions de l'intensité (incidence annuelle) et du calendrier (dates de démarrage, de maximum, de fin) des épidémies sont donc complexes ; c'est pourquoi elles ont pris de plus en plus d'ampleur dans le contexte de la transition et du changement climatiques. Avant AMMA, seules quelques études empiriques avaient porté sur le lien entre climat et épidémiologie (Thomson et al., 2006). En outre, la variable « aérosols » n'avait été que peu prise en compte. Enfin, les données épidémiologiques n'avaient jusqu'ici été que très peu exploitées dans la communauté des géophysiciens. La combinaison de ces trois points a permis des avancées importantes. Cet article permet donc de donner à titre d'éclairage quelques exemples de résultats sur les liens climat-santé, parmi d'autres travaux réalisés dans le cadre AMMA, en particulier ceux portant sur la fièvre de la vallée du Rift au Sénégal (Ndione et al., 2005) ou encore les travaux sur la pollution urbaine dans les mégacités africaines (Liousse et Galy-Lacaux, 2010).

\section{Le paludisme, une pathologie de la saison des pluies}

La relation entre le paludisme, le climat (via les précipitations et la température) et l'environnement (mares pérennes ou temporaires, végétation) est suspectée depuis longtemps, comme l'indique l'étymologie latine de son nom, paludis, marais. Il s'agit d'une maladie transmise par un moustique du genre Anopheles, dont la femelle a besoin d'un « repas » sanguin pour assurer la maturation de ses œufs, avant de les pondre dans l'eau. Les anophèles ont besoin pour survivre de conditions climatiques et environnementales spécifiques (figure 1), qui diffèrent selon les espèces et l'emplacement géographique. On sait aussi notamment que le développement du parasite est très sensible aux conditions de température.

La première description par Ronald Ross en 1897 de l'implication du moustique dans la transmission a été à l'origine des premières campagnes de lutte anti-vectorielle et de l'élimination du paludisme dans de nombreuses régions du monde. Mais il ne faut pas oublier que cette élimination n'est que très récente : 1'Europe n'a été déclarée indemne qu'en 1975 (Reiter, 2004) et une réapparition de $P$. vivax est évoquée en Grèce. Malgré les efforts des programmes de lutte, le nombre de décès à cause du paludisme a été estimé à 665000 en 2010. Avec 216 millions de cas en 2010 (dont $81 \%$ en Afrique subsaharienne), il reste endémique dans 106 pays (OMS, 2011). Une des principales causes de l'échec des programmes de lutte est la forte hétérogénéité de la dynamique de transmission du paludisme, dans le temps et dans l'espace. L'origine de cette hétérogénéité est multiple. Différentes espèces d'Anopheles peuvent intervenir, chaque espèce ayant un comportement différent. Par
Figure 1 - Gîte temporaire d'Anopheles gambiae (principal vecteur du paludisme dans cette zone) en bordure de la ville de Bandiagara au Mali. (C) Jean Gaudart)

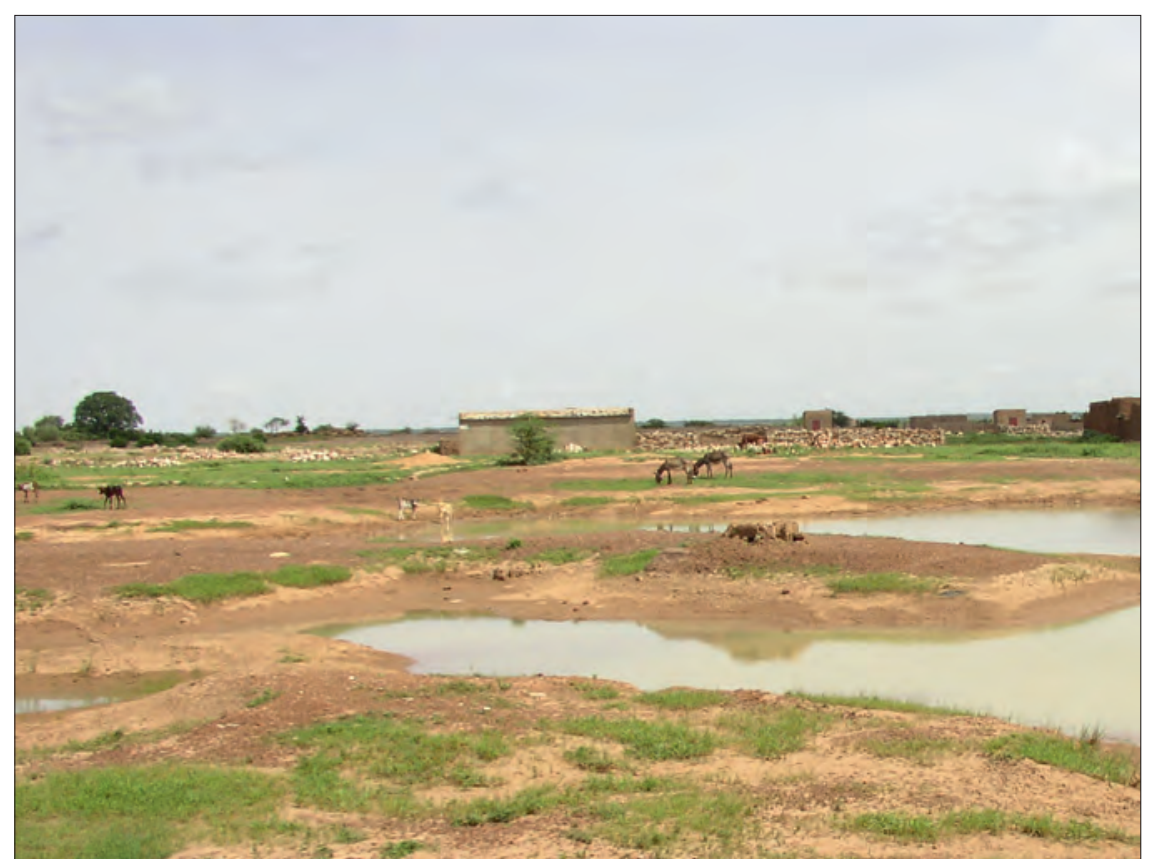


exemple et schématiquement, les larves d'Anopheles gambiae s1 se développent plus facilement en eau peu profonde, chaude et pauvre en végétation, alors que les larves d'Anopheles funestus s1 préfèrent les eaux plus fraîches comportant plus de végétation. Les comportements humains jouent également un rôle important, au travers notamment de la modification de l'environnement (urbanisation, agriculture, barrages, creusement de puits, briqueteries, etc. ; Afrane et al., 2004 ; Krefis et al., 2011), et des migrations qui assurent le brassage de souches parasitaires. Le parasite lui-même évolue, avec l'apparition régulière de souches résistantes aux médicaments.

On peut se rendre compte de cette hétérogénéité en regardant l'incidence du paludisme sur plusieurs années. Un travail en collaboration avec les équipes maliennes du MRTC (Malaria Research and Training Center), a permis de mesurer l'incidence du paludisme de mai 1996 à février 2001, dans la ville de Bancoumana (figure 2a), située à $60 \mathrm{~km}$ au sud-ouest de Bamako au Mali (Gaudart et al., 2009). L'analyse de cette série temporelle a montré d'abord une saisonnalité de la transmission, qui se fait essentiellement en saison des pluies, de fin juin à début septembre. Pour rechercher un facteur explicatif climatique et en l'absence de données de précipitation fiables, nous avons utilisé l'indice de végétation (NDVI), comme indicateur des précipitations. Il a été calculé comme la différence normalisée des réflectances corrigées des canaux proche infrarouge et visible pour une série d'images satellites
NOAA (1996-2001). Cet indice, qui permet de quantifier par télédétection l'activité chlorophyllienne de la végétation (Rouse et al., 1974), est employé ici comme substitut pour analyser les impacts de la variabilité des précipitations. À l'aide d'une modélisation mathématique, nous avons montré que la saisonnalité de l'incidence est presque totalement expliquée par le climat. Dans cette région, il existe un délai de 15 jours entre l'augmentation du NDVI et l'augmentation de l'incidence palustre, qui s'explique par la durée du développement des larves d'anophèles. Nous avons également montré qu'il existe un seuil de NDVI $(0,361$ dans cette région) au-delà duquel la transmission s'accélère. Bien sûr, ce seuil peut varier en fonction du contexte local. À Bancoumana, la transmission peut se poursuivre même pendant la saison sèche, mais avec un impact moins important. Cette persistance montre que la pluie n'est pas le seul facteur à l'origine de la transmission et d'autres facteurs restent encore à identifier. Par exemple, il existe des zones de maraîchage conservant une humidité suffisante pour la survie des vecteurs et pour assurer la transmission en saison sèche. De plus, nous avons retrouvé des larves d'anophèles pendant la saison sèche dans les puits.

Dans la situation étudiée (figure 2a), la courbe des incidences rend compte également d'un autre phénomène : il existe, dans ce village, une tendance à la décroissance de l'incidence palustre passant en moyenne de $60 \%$ de contamination en 1996 à $35 \%$ en 2011 . Cette tendance s'explique par la présence en

Figure 2 - a) Évolution de l'incidence palustre (courbe rouge) de mai 1996 à janvier 2001, ville de Bancoumana (Mali). La courbe verte représente l'évolution de l'indice de végétation (NDVI) obtenue par imagerie satellitaire. Les courbes bleues représentent la modélisation statistique de la saisonnalité (et son intervalle de confiance à $95 \%$ en pointillé). b) Cartographie des cas de paludisme, Bancoumana, août 1999. Chaque point représente une concession (groupe d'habitations appartenant à une grande famille). L'échelle de couleur représente le pourcentage de cas dans chaque concession. Les risques relatifs sont indiqués dans chaque zone. Le découpage en zones de risque homogène (traits noirs) a été réalisé à l'aide de l'algorithme SPODT.

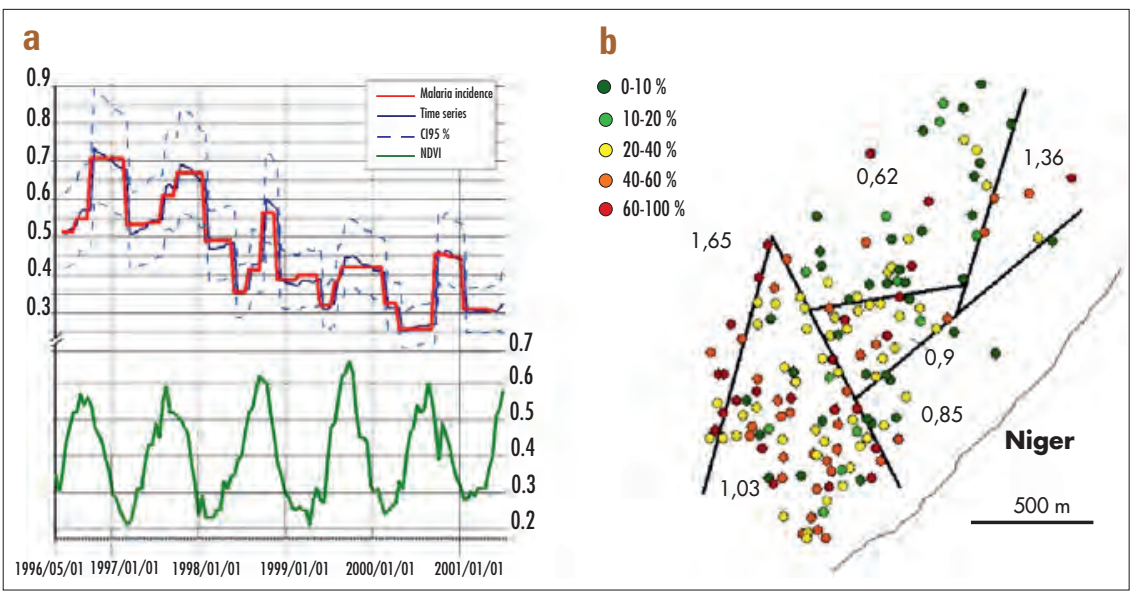

continu d'une équipe médicale sur place. En effet, le traitement des personnes malades est utile non seulement pour leur propre guérison, mais également dans un but épidémiologique : un traitement rapide rompt la chaîne de transmission.

Il faut cependant se garder d'inférer à l'ensemble des régions ce que l'on observe sur une seule ville. En effet, des conditions environnementales très différentes peuvent être à l'origine d'une transmission pérenne, en particulier en milieu équatorial, ou d'une transmission épidémique ponctuelle, en particulier en milieu subdésertique (Koenraadt et al., 2003 ; Krefis et al., 2011). La transmission peut même être variable à l'échelle d'une ville. L'analyse de l'hétérogénéité du risque palustre et des facteurs climatiques et environnementaux est une condition indispensable pour l'élaboration de stratégies de luttes durables et adaptées au contexte local (comme le recommande l'initiative MALERA - Malaria Eradication Research Agenda ; Alonso et al., 2011). Pour mettre en évidence l'hétérogénéité spatiale du risque palustre à Bancoumana, nous avons recherché un découpage du village en zones de risques différents, à l'aide de l'algorithme SPODT (Spatial Oblique Decision Tree ; Gaudart et al., 2005), représentant ainsi cette hétérogénéité du risque. Nous avons pu mettre en évidence six zones géographiques de risque différent (degrés de signification $p=0,047$ ), figure $2 b$, avec notamment une zone à l'ouest avec un risque relatif de 1,65 et une seconde zone (risque relatif de 1,36) au nord-est du village. Ces 2 zones à risque élevé comportent des particularités environnementales. À l'ouest, la roche affleure et l'eau stagne dès les premières pluies. Au nord-est se trouve une petite briqueterie. Ces particularités favorisent le développement de gîtes larvaires dès les premières pluies et sont donc à l'origine du risque élevé.

Une autre étude nous a amenés à estimer le risque palustre dans la ville de Bandiagara, à $800 \mathrm{~km}$ au nord-est de Bamako au Mali (Dessay et al., 2010). À l'aide de l'imagerie satellitaire à haute résolution cette fois (Quick-Bird, Spot), ont été mesurées la densité d'habitat et la distance aux principaux gîtes de pontes. En combinant ces 2 facteurs, sociétal (vulnérabilité) et environnemental (aléa), nous avons mis en évidence différentes zones à risque à l'échelle de la ville (figure 3 ). Il faut noter que ces travaux nécessitent des études de terrain avec un suivi important des 


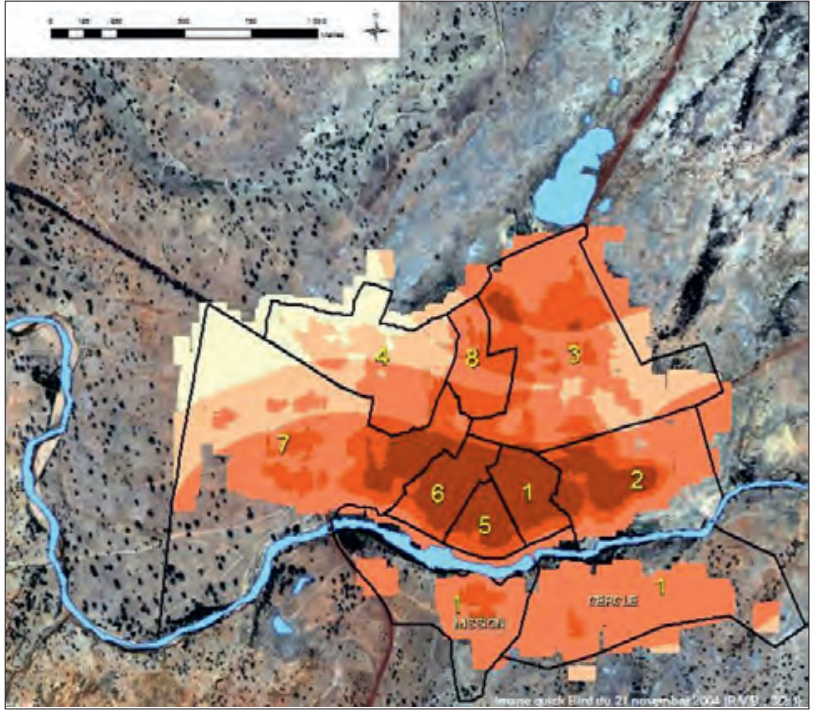

populations, mais aussi le développement de nouveaux outils spécifiques, en particulier de télédétection et d'analyse statistique. Notre approche est multidisciplinaire et associe climatologues, géographes, épidémiologistes, parasitologistes, spécialistes de santé publique et de sciences humaines, afin de pouvoir comprendre au mieux les relations entre paludisme, climat et société. En terme de santé publique, cette connaissance permet d'envisager des stratégies de luttes fondées sur des mesures de contrôles différentes selon le contexte, adaptées à l'environnement et aux populations locales (moustiquaires imprégnées d'insecticides, spray intradomiciliaires, gestion de l'environnement, diagnostic et traitement rapide des malades, traitement curatif intermittent saisonnier, etc.).

\section{Le choléra, une pathologie climato-sensible?}

En Afrique de l'Ouest, les relations entre le choléra, le climat et l'environnement ont été peu étudiées. Cette maladie est volontiers considérée comme endémique dans de nombreuses régions côtières, depuis le golfe de Guinée jusqu'aux frontières du Sénégal. Mais le germe responsable de la maladie n'est pas originaire d'Afrique : son importation a eu lieu à Conakry en 1970 . Une fois importé, le choléra peut bénéficier des effets des précipitations comme à Conakry, où les épidémies sont d'autant plus marquées que les premiers cas surviennent précocement après le début de la saison des pluies, dans les provinces côtières de Guinée-Bissau ou en SierraLeone et au Liberia (GTFCC, 2010).
Figure 3 - Ville de Bandiagara, estimation qualitative du risque palustre en fonction de la densité de population et de la distance aux gîtes d'Anophèles. En jaune, l'estimation est minimale, nous sommes à plus de 600 mètres d'étendues d'eau favorables au développement d'anophèles. En rouge, l'exposition est maximale.

Dans ces pays, les pluies saisonnières peuvent favoriser le débordement des latrines et ainsi contaminer les puits. Au début des années 1990, les épidémies de choléra qui ont frappé la Côte d'Ivoire, le Ghana, le Togo, le Bénin et le Nigeria étaient significativement corrélées avec les températures de surface de l'océan Indien (Constantin de Magny et al., 2007). Ces anomalies climatiques globales peuvent provoquer localement des inondations ellesmêmes tenues pour responsables de certaines épidémies de choléra, par exemple lors d'un pic épidémique de choléra à Dakar (Manga et al., 2008). En fait, beaucoup d'autres facteurs interviennent et certaines épidémies de choléra en Afrique semblent clairement avoir été causées par l'introduction de nouvelles souches de $V$. cholerae, comme en Guinée-Bissau en 1994 ou au Sénégal l'année suivante. Ces souches importées peuvent avoir bénéficié de conditions climatiques et sociales favorables comme l'atteste la plus grande incidence du choléra dans les zones urbaines les plus défavorisées où l'accès à l'eau et l'évacuation des eaux usées font défaut. Outre les régions côtières d'Afrique de l'Ouest, les épidémies de choléra frappent régulièrement les populations du Sahel (Piarroux et Faucher, 2012). Même si les épidémies sont souvent reliées à la saison des pluies, les relations entre le choléra et la pluie sont particulièrement compliquées au Sahel. Au Mali, la plupart des épidémies de ces vingt dernières années ont été contemporaines de la saison des pluies, touchant préférentiellement les régions baignées par le fleuve Sénégal (la région de Kayes en 1996 et 2005) et le fleuve Niger (Mopti, Ségou, Tombouctou), voir Dao et al., 2009. L'épidémie qui sévit en 2005 à Ouagadougou, au Burkina Faso, survint aussi en saison des pluies. Plus en aval, le long du fleuve Niger, les relations entre climat et choléra apparaissent encore plus complexes : ces dernières années, le Niger a connu plusieurs épidémies meurtrières, dont certaines étaient associées à des épisodes de sècheresse (2004) et d'autres à des épisodes de pluies abondantes (2006) (GTFCC, 2010). Plusieurs études descriptives ont montré une association possible entre la saison sèche et le choléra au Sahel, comme en 1970 à Mopti au Mali, ou durant les sècheresses des années 1970 et en 1984-85. Les concentrations de population autour des ressources en eau encore disponibles et la surpopulation dans des campements de personnes déplacées peuvent expliquer ce phénomène (Tauxe et al., 1988).

Les précipitations et les facteurs environnementaux jouent donc un rôle important dans les épidémies de choléra en Afrique de l'Ouest. Cependant, de même que la géographie de cette région du monde est diverse, l'épidémiologie du choléra se caractérise par une hétérogénéité marquée, certaines épidémies étant manifestement favorisées par la pluie, d'autres survenant parfois aux mêmes endroits, par des épisodes de sècheresse, les populations se regroupant autour de rares points d'eau contaminés. Il est donc primordial de continuer les recherches sur le lien entre climat et choléra. En termes de santé publique, la prévention et la gestion des épidémies de choléra ne peut passer que par une connaissance précise du terrain et des facteurs favorisant l'émergence et l'expansion des épidémies dans cette région.

\section{La méningite, une pathologie de la saison sèche}

\section{Intensité des épidémies}

L'intensité (incidence annuelle) des épidémies de méningite à Neisseria meningiditis en Afrique sahélienne est liée à hauteur de $25 \%$ au climat. Yaka et al. (2008) ont mené une analyse statistique multidimensionnelle entre incidence annuelle de méningite issue de la base de données de surveillance de l'Organisation mondiale de la santé (OMS) en Afrique, des ministères de la Santé du Burkina Faso et du Niger, et des variables climatiques issues des réanalyses américaines du NCEP-NCAR (Kanamitsu et al., 2002) sur la période 19692005. Les coefficients de corrélation entre les valeurs mensuelles de chaque paramètre atmosphérique et le logarithme 


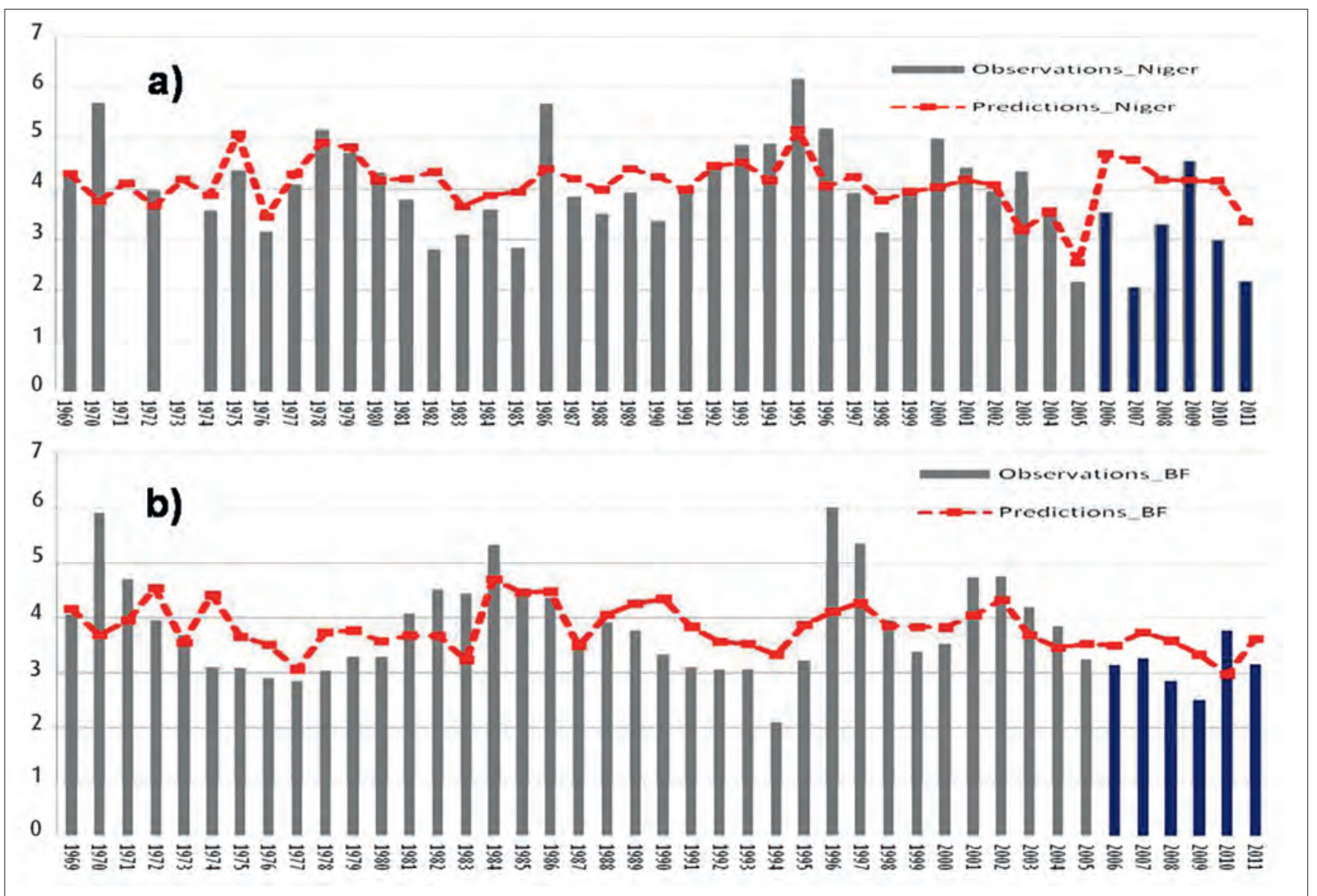

Figure 4 - Comparaisons entre les logarithmes népériens des incidences annuelles de méningite observée (barres verticales) et simulée (courbes) au Niger (a) et au Burkina Faso (b) de 1969 à 2011. La période 1969 à 2005 a été utilisée pour élaborer le modèle. De 2006 à 2011 (en bleu foncé), le modèle a été employé dans un cadre opérationnel, pluridisciplinaire, pour la prédiction des épidémies de méningite au Burkina Faso.

népérien de l'incidence annuelle de méningite ont d'abord été calculés pour les mois précédant les périodes épidémiques, c'est-à-dire d'octobre à janvier (significativité au seuil de confiance de $95 \%$ basée sur le test de Monte-Carlo). Cette analyse a permis de sélectionner un groupe de prédicteurs afin d'élaborer un modèle de régression linéaire multiple pas à pas permettant de simuler l'incidence annuelle de méningite. La robustesse du modèle est attestée par la méthode de validation croisée. Parmi les paramètres climatiques étudiés au Niger, les auteurs ont pu retenir la composante méridienne du vent des mois de novembre et de décembre. Au Burkina Faso, a uniquement été retenue la composante méridienne du vent du mois d'octobre. Les résultats du modèle du Burkina Faso sont présentés pour exemple en figure 4 .

Les corrélations entre observations et simulations sont très significatives au Niger $(\mathrm{r}=0,62)$ et au Burkina Faso $(\mathrm{r}=0,42)$. Le coefficient de validation croisée est de 0,50 pour le modèle conçu pour le Niger et de 0,33 pour celui du Burkina Faso ; ce qui signifie qu'environ un quart de la variance totale de l'incidence annuelle de méningite au Niger peut être expliquée par la variabilité des paramètres climatiques en saison sèche.

Malgré un coefficient de corrélation en validation croisée moins élevé, le modèle s'avère significatif pour prévoir les incidences annuelles de méningite au Burkina Faso pour les années post-2005. Ce qui confirme sa robustesse. Ainsi, l'augmentation des incidences annuelles de méningite dans ces deux pays est liée au renforcement des vents secs venant du nord, plus connus sous le nom d'harmattan, pendant les mois de novembre et décembre au Niger, et au mois d'octobre au Burkina Faso. Ces modèles sont encore perfectibles, moyennant l'adjonction d'autres prédicteurs potentiels (environnementaux tels que les aérosols désertiques, socio-démographiques, économiques et biologiques). Toutefois, les résultats montrent que ces modèles contribuent significativement à l'élaboration d'un système intégré de surveillance et d'alerte précoce des épidémies de méningite en Afrique soudano-sahélienne.

\section{Démarrage des épidémies de méningite}

Les épidémies de méningite démarrent en février pour disparaître au mois de mai en Afrique sub-saharienne. En effet, la partie soudano-sahélienne de l'Afrique est soumise à l'alternance d'une saison sèche en hiver, dominée par des vents d'harmattan, et une saison humide qui démarre au printemps pour être maximale en été avec la mise en place de la mousson. Le contexte climatique caractéristique de l'hiver des latitudes subtropicales présente des conditions favorables au développement de la méningite : alors que la sécheresse et les vents forts, chargés de poussières, peuvent stimuler l'invasion du méningocoque en lésant directement la barrière muqueuse ou en inhibant les défenses immunitaires de surface, l'humidité du printemps et des latitudes guinéennes réduit considérablement l'incidence de la méningite (Chippaux et al. 2002). Sultan et al. (2005b) ont croisé les recensements hebdomadaires de l'OMS de cas et de décès liés à la méningite au Mali pour la période 1994-2002 et les variables 


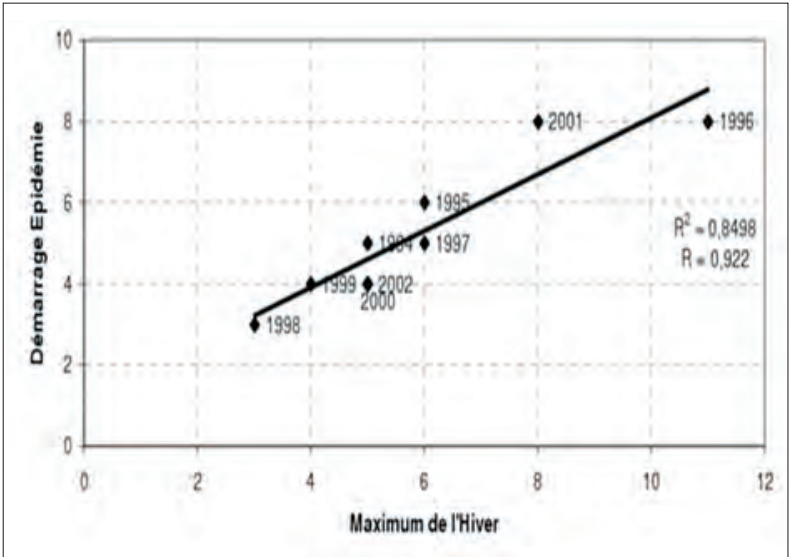

Figure 5 - Relation entre la semaine du démarrage de l'épidémie au Mali (ordonnées) et la semaine du maximum de I'hiver (abscisses). Figure adaptée de Sultan et al. (2005b).

atmosphériques issues des réanalyses NCEP-NCAR. À l'échelle saisonnière, la construction d'indices atmosphériques à partir de l'humidité et du vent de surface a montré un synchronisme de la dynamique été/hiver du climat avec la progression des épidémies de méningite, dont le démarrage coïncide avec le maximum de l'hiver (date à laquelle la circulation d'harmattan est la plus forte) en moyenne entre le 7 et le 15 février (figure 5).

Plus remarquable encore, les auteurs trouvent une corrélation très forte de 0,92 sur les années de 1994 à 2002 traduisant le fait que le moment du maximum de l'hiver explique plus de $85 \%$ des dates de démarrage des épidémies de méningite au Mali, ce qui signifierait qu'un hiver précoce (respectivement tardif) au Mali est associé à un démarrage précoce (respectivement tardif) des épidémies de méningite.

\section{Fin des épidémies de méningite}

Des résultats provenant de l'analyse de jeux de données différents et de méthodologies variées montrent que la fin des épidémies de méningite est liée à l'arrivée de la mousson africaine. Depuis 2002, une collecte d'échantillons de liquide céphalo-rachidien de patients suspects d'être atteints de méningite a été instaurée entre les hôpitaux, les centres de santé primaires et le Centre de recherche médicale et sanitaire au Niger. En 2006, Jeanne et ses collaborateurs ont mis en place une analyse conjointe des données confirmées de méningites, quasi exhaustives et très fiables (Boisier et al., 2007) et des données hydrométéorologiques acquises in situ à Banizoumbou au Niger durant la Special Observation Period (SOP) d'AMMA (Rajot et al., 2008). Cette année là eut lieu une épidémie de méningite à méningocoque de sérogroupe $\mathrm{X}(\mathrm{NmX})$ qui a débuté par Niamey. Comme aucune épidémie n'a eu lieu les années précédentes et comme il n'existe aucune vaccination contre ce méningocoque, les résultats sont plus robustes. L'exploitation de ces données de 2006 a permis de montrer que la chute brutale du nombre de cas de méningite à NmX était contemporaine d'un changement important de direction du vent, qui correspond à l'installation du flux de mousson dont les vents d'origine marine sont beaucoup plus humides (Jeanne, 2007). Plus récemment, Martiny et Chiapello (2012) ont utilisé les recensements hebdomadaires de l'OMS de cas et de décès liés à la méningite au Niger, Mali et Burkina Faso pour la période 20042009 ainsi que les données d'humidité issus du réseau de mesures photométriques AERONET. Les résultats de cette étude au Niger (figure 6) montrent que l'augmentation de l'humidité (semaines 13 à 16, fin mars-début avril) coïncide avec une phase de diminution du nombre de cas sur la même période et signe ensuite l'arrêt du cycle saisonnier de la méningite (que les années soient épidémiques ou non), en semaine 16-17 (miavril). Les résultats obtenus au Burkina Faso et au Niger, non montrés ici, sont similaires.

\section{Variabilité du nombre de cas}

L'intensité ainsi que le calendrier des épidémies au Sahel semblent très liés aux variables climatiques vent et humidité. En outre, il semble que la variabilité du nombre de cas entre le démarrage et le maximum du cycle saisonnier de la méningite soit très liée à la variabilité des événements de poussières désertiques, comme suspecté par Martiny et al. (2007), dont les caractéristiques ont été étudiées dans AMMA par le biais notamment de mesures de terrain acquises sur le Sahelian Dust Transect situé à $13^{\circ} \mathrm{N}$ (Rajot et al., dans ce numéro, p. 33-40). Martiny et Chiapello (2012) ont utilisé les recensements hebdomadaires de l'OMS de cas et de décès liés à la méningite au Niger, Mali et Burkina Faso pour la période 2004-2009 (cœur du programme AMMA) ainsi que les données aérosols issues du réseau de mesures photométriques AERONET. En effet, les aérosols désertiques semblent être un facteur déclenchant (Mueller et Gessner, 2010), ce qui complète la théorie de Sultan et al. (2005b) selon laquelle le démarrage du cycle saisonnier de la méningite est en phase avec le maximum de l'hiver. Ainsi, et cela confirme les travaux de Jeanne (2007) spécifiques à l'année 2006 au Niger, une augmentation du contenu en aérosols précède systématiquement une augmentation du nombre de cas de méningite, et ce avec un certain temps de réponse. Ce temps de réponse, en moyenne de 2 semaines au Niger, Burkina Faso et Niger, semble correspondre au temps d'incubation de la maladie (Agier et al., 2012).

En effet, les auteurs observent au Niger (figure 6) une augmentation du contenu en aérosols précédant chaque

Figure 6 - Cycle saisonnier moyen standardisé de méningite, superposé aux cycles des aérosols et de l'humidité spécifique au Niger sur la période 2004-2009. Adapté de Martiny et Chiapello (2012)

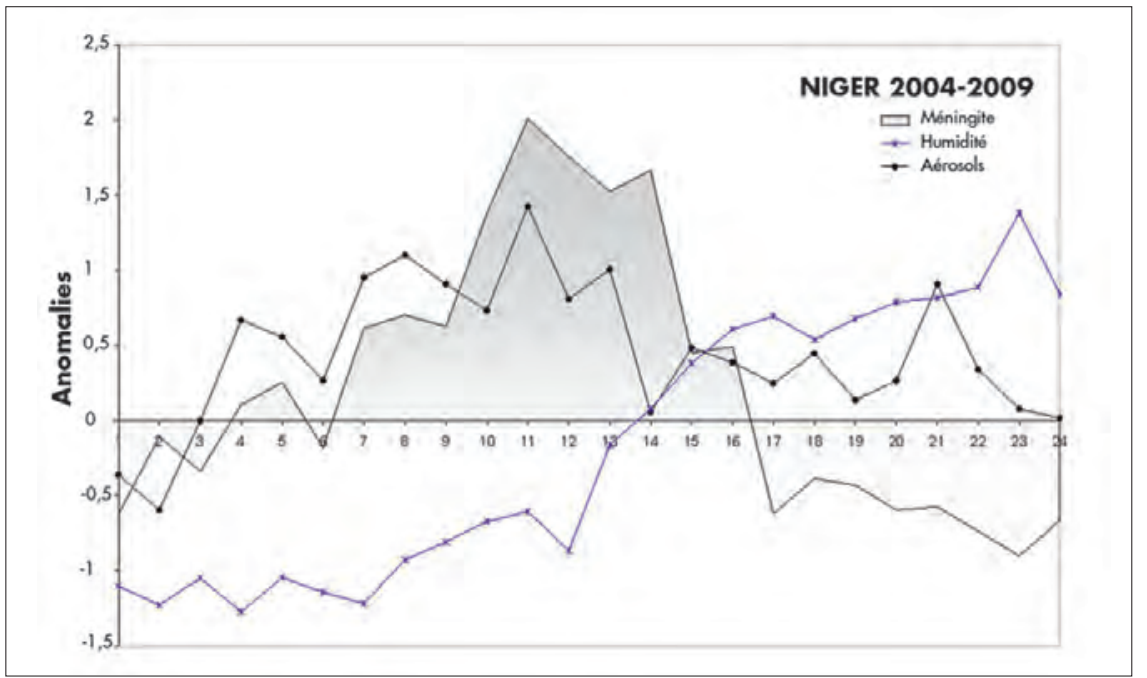


augmentation du nombre de cas de méningite du démarrage (semaine 4) au dernier pic (semaine 14) de son cycle saisonnier. Ces résultats ont d'ailleurs été retrouvés par le biais de données issues des missions spatiales (Eymard et al., dans ce numéro, p. 80-89). Cette relation entre aérosols et méningite semble s'interrompre lors de l'augmentation de l'humidité liée à l'arrivée de la mousson. En effet, le cycle saisonnier des aérosols s'arrête bien plus tardivement que celui des méningites (semaine 24, mi-juin), mais les événements de poussières survenant après la semaine 13 (début de la phase d'augmentation de l'humidité) ne semble plus jouer de rôle sur le nombre de cas de méningite.

\section{Conclusions et perspectives}

Malgré des connaissances anciennes sur la relation climat-paludisme, les travaux récents réalisés notamment par AMMA montrent que les interactions homme-climat-environnement-parasite sont complexes et nécessitent des approfondissements à l'échelle des sociétés. Actuellement, les programmes de luttes les plus efficaces sont ceux qui combinent plusieurs actions, chacune devant être adaptée au contexte local, environnemental et social. En effet, l'association de plusieurs actions, notamment du traitement après un diagnostic rapide, de l'utilisation de moustiquaires imprégnées (en saison des pluies) et d'une gestion de l'environnement (comblement de gîtes, rigoles d'évacuation des eaux de pluies...) permettrait d'accélérer la lutte contre le

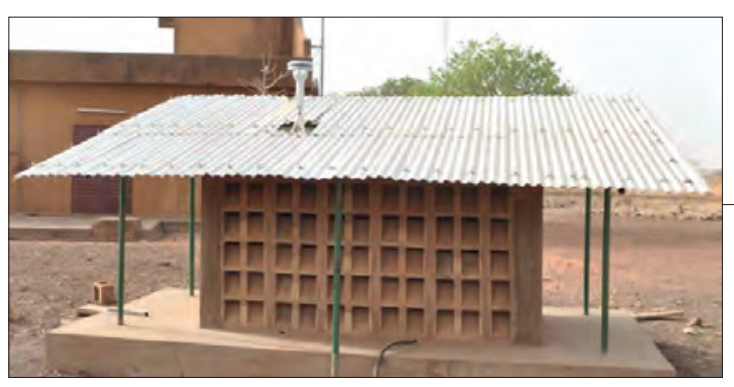

Figure 7 - Implantation d'un nouvel instrument de mesures d'aérosols à la station météorologique de Dédougou au Burkina Faso, en mars 2012.

(c) R. Belleville, Terra Deserta Expéditions)

Par ailleurs, il est crucial pour la suite que plus de données de terrain sur les aérosols soient acquises

paludisme. Mais il faut aussi poursuivre la quantification de l'effet relatif de chaque action à une échelle locale, tenant ainsi compte de l'hétérogénéité de la transmission. Concernant le choléra, il n'existe que très peu d'études sur la dynamique des épidémies au Sahel. Les effets de la pluie et de la sècheresse sur leur démarrage et leur diffusion au Sahel doivent être mieux compris, afin de mettre en place des actions de prévention ciblées.

Au début d'AMMA, peu d'études quantitatives existaient sur le lien entre climat et méningite. Aujourd'hui, les variables climatiques clés pour les épidémies de méningite ont été identifiées à l'échelle nationale au Burkina Faso, au Niger et au Mali, pays touchés à des degrés différents par les épidémies. Au vu des connaissances actuelles, la construction d'indices climatiques et d'indices d'aérosols offre un potentiel important en matière de prévision et de mise en place d'études de risque de la maladie en Afrique. Les données climatiques issues des réanalyses ou des modèles climatiques, ainsi que les données d'aérosols issues des modèles d'émission au pas de temps hebdomadaire, présentent donc un intérêt évident pour les services de santé afin de prévoir la date de démarrage de ces épidémies. dans une optique de validation (modèles ou données satellites). Ainsi, un nouvel instrument de mesures d'aérosols a été implanté à Dédougou au Burkina Faso depuis mars 2012 sur la base d'un partenariat entre le Centre de recherches de climatologie et les Services de la météorologie du Burkina Faso (figure 7).

Enfin, depuis 2008, chaque année en début de période épidémique, se retrouvent, dans le cadre d'une collaboration étroite entre les Services de la météorologie et ceux de la santé publique du Burkina Faso, les techniciens de ces institutions afin d'élaborer un bulletin commun sur la prévision de la tendance de l'incidence annuelle de méningite au Burkina Faso et au Niger. Ceci, en exploitant les résultats des modèles statistiques développés par Yaka et al. (2008) ainsi que des informations d'analyses épidémiologiques sur la méningite. Ces prévisions pourraient ainsi apporter une contribution à la prise de décision pour les campagnes de vaccination et l'approvisionnement des tests diagnostiques rapides, au lit du patient et thérapeutiques (Chanteau et al., 2007 ; Cuevas et al., 2007). Il est important de mentionner que ces résultats opérationnels concrétisent une étape importante dans la mise en place de systèmes d'alerte précoce pour les épidémies de méningite. 